\title{
Precision Muon Capture at PSI
}

\author{
Peter Kammel ${ }^{* \dagger}$ \\ Department of Physics and Center for Experimental Nuclear Physics and Astrophysics, \\ University of Washington, Seattle \\ E-mail: pkammeleuw. edu
}

The muon capture program at PSI is reviewed. Its focus is on hydrogen and deuterium, where theoretical predictions based on first principle calculations within chiral effective field theory are available. The completed analysis of the MuCap experiment unambiguously determines the muon capture rate on the proton and derives the pseudoscalar coupling of the nucleon, leading to a stringent test of QCD at low energies. The MuSun experiment determines a poorly known low energy constant from muon capture on the deuteron, which is needed to calculate fundamental astrophysics reaction, such as solar pp fusion.

The 7th International Workshop on Chiral Dynamics,

August 6 -10, 2012

Jefferson Lab, Newport News, Virginia, USA

\footnotetext{
${ }^{*}$ Speaker.

${ }^{\dagger}$ Representing the MuCap and MuSun Collaborations
} 


\section{Scientific Motivation}

We are now at the confluence of two exciting developments in the field of muon capture [1]. Based on the novel "active target" technique developed by our collaboration, experiments on hydrogen and the lightest nuclei are approaching sub-percent precision, surpassing the precision of previous generations of experiments by nearly an order of magnitude. At the same time, effective field theories (EFTs), based on the symmetries of the underlying fundamental theory of quantum chromodynamics (QCD), have been systematically constructed to calculate electro-weak interactions of nucleons and few-body systems. Thus simple nuclear systems become amenable to rigorous QCD-based calculations.

Our scientific program consists of the following experiments at the high intensity muon beam lines of the Paul Scherrer Institute, Switzerland. The MuCap experiment is a precision measurement of muon capture on the proton to determine the pseudoscalar form factor $g_{P}$ in the axial current of the nucleon. We present the result of the analysis of the experiment's complete data set, which provides a basic test of QCD at low energies. The ongoing MuSun experiment extends these measurements to muon capture on the deuteron. MuSun will determine the sole unknown lowenergy constant $\hat{d}_{R}$ entering the EFT calculation of weak interactions in the two-nucleon system. It will be a benchmark result for extending the EFT method to more complicated few-body processes, and for calibrating the calculations of related astrophysical processes of fundamental importance. In the context of this program we also summarize the final result of the MuLan experiment. It measures the free muon lifetime and determines the strength of the weak interaction, the Fermi constant $\mathrm{G}_{F}$, with unprecedented precision.

\subsection{Free muon lifetime}

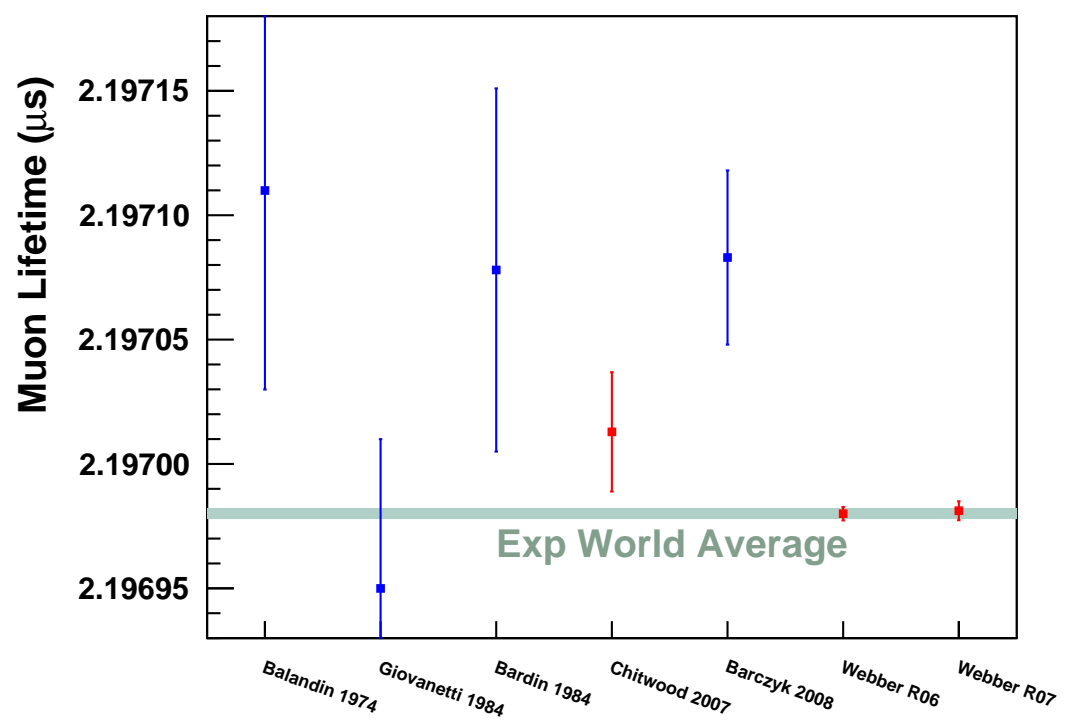

Figure 1: Free muon lifetime measurements. Red data points denote MuLan results: commissioning run (Chitwood 2007), measurement with ferromagnetic target (Webber R06) and quartz crystal target with external field (Webber R07). 
The Fermi constant $\mathrm{G}_{F}$ is best determined via the relation

$$
\lambda_{\mu^{+}}=\frac{G_{F}^{2} m_{\mu}^{5}}{192 \pi^{3}}\left(1+\sum_{i} \Delta q^{(i)}\right) .
$$

On the left hand side $\lambda_{\mu^{+}}=\frac{1}{\tau_{\mu}}$ is the free muon decay rate expressed as inverse of the muon lifetime. The expression on the right hand side includes $G_{F}$ and $m_{\mu}$, the muon mass, while $\sum \Delta q^{(i)}$ describes the phase space and higher QED corrections[2]. Up to 1999 the precision of this evaluation of $G_{F}$ was limited to $15 \mathrm{ppm}$ by the unknown second-order QED term. Calculation[2] and subsequent work reduced the theoretical uncertainty to $0.14 \mathrm{ppm}$. At that time the measurements of $\tau_{\mu}$ contributed the dominant $9 \mathrm{ppm}$ uncertainty to $G_{F}$. As seen in Fig. 1, this stimulated an experimental program to improve the experimental lifetime dramatically. Recently, this goal has been achieved by the MuLan collaboration [3, 4, 5], which now dominates the world average. The resulting lifetime and decay rates are measured at the $1 \mathrm{ppm}$ level:

$$
\begin{aligned}
\tau_{\mu}^{P D G} & =2.1969811 \pm 0.0000022 \mu s \\
\lambda_{\mu^{+}}^{P D G} & =455170.05 \pm 0.46 s^{-1}
\end{aligned}
$$

From its $\tau_{\mu}$ result MuLan [5] derives

$$
G_{F}(\text { MuLan })=1.1663787(6) \times 10^{-5} \mathrm{GeV}^{-2}(0.5 \mathrm{ppm}) .
$$

This represents a thirty-fold improvement over the 1999 PDG value, reflecting the combined theoretical and experimental progress on this fundamental constant over the last decade.

\subsection{Muon Capture and Nucleon Form Factors}

Muon capture on the proton,

$$
\mu^{-}+p \rightarrow n+v_{\mu}
$$

is a basic charged-current weak process. It proceeds with a rate $\Lambda_{S}$ after a muonic hydrogen atom has been formed in its lower singlet hyperfine state. For the low momentum transfer $q_{0}^{2}=-0.88 m_{\mu}^{2}$ in process (1.4), the Standard Model electroweak interaction reduces to an effective Fermi interaction between the lepton current $L_{\mu}$ and the hadronic current $J_{\mu}$. While $L_{\mu}$ retains its simple $\gamma_{\mu}\left(1-\gamma_{5}\right)$ structure, the hadronic current $J^{\mu}=V^{\mu}-A^{\mu}$ between nucleon states is modified by QCD, as expressed in a model-independent way by the introduction of form factors (neglecting suppressed $2^{\text {nd }}$ class terms)

$$
\begin{aligned}
& <n\left|V^{\mu}\right| p>=\bar{u}_{n}\left[g_{V}\left(q^{2}\right) \gamma^{\mu}+\frac{i g_{M}\left(q^{2}\right)}{2 m_{N}} \sigma^{\mu v} q_{v}\right] u_{p}, \\
& <n\left|A^{\mu}\right| p>=\bar{u}_{n}\left[g_{A}\left(q^{2}\right) \gamma^{\mu} \gamma^{5}+\frac{g_{P}\left(q^{2}\right)}{m_{\mu}} q^{\mu} \gamma^{5}\right] u_{p} .
\end{aligned}
$$

Here $m_{N}$ is the nucleon masses and $q$ is the momentum transfer. The vector and magnetic form factors $g_{V}\left(q^{2}\right)$ and $g_{M}\left(q^{2}\right)$ are determined by the conserved vector current hypothesis and electron scattering experiments. The precise value of the axial form factor $g_{A}\left(q^{2}\right)$ is still subject to ongoing 
discussions. Its current PDG value is $g_{A}^{\mathrm{PDG}}(0)=1.2701 \pm 0.0025$ [6], although it has been advocated [7] that $g_{A}(0)$ should be increased to 1.275 , to be consistent with recent measurements of the neutron $\beta$-decay asymmetry $[8,9]$ and the revised neutron lifetime [6]. Nevertheless, compared to the pseudoscalar form factor $g_{P}$, the first three form factors are well known and contribute only $0.4 \%$ uncertainty to $\Lambda_{S}$. Thus the goal of the MuCap experiment is to determine $g_{P} \equiv g_{P}\left(q_{0}^{2}\right)$ with precision by measuring the rate for process (1.4).

Chiral effective field theory $[10,11]$ allows a robust and rather precise calculation of

$$
g_{P}^{\text {theory }}=\frac{2 m_{\mu} g_{\pi N N} F_{\pi}}{m_{\pi}^{2}-q^{2}}-\frac{1}{3} g_{A}(0) m_{\mu} m_{N} r_{A}^{2}=8.26 \pm 0.23 \text {. }
$$

The good convergence of this result has been verified up to two-loop order [12]. Ab-initio calculation within lattice QCD [13,14] are ongoing, but have not yet reached the precision of EFT.

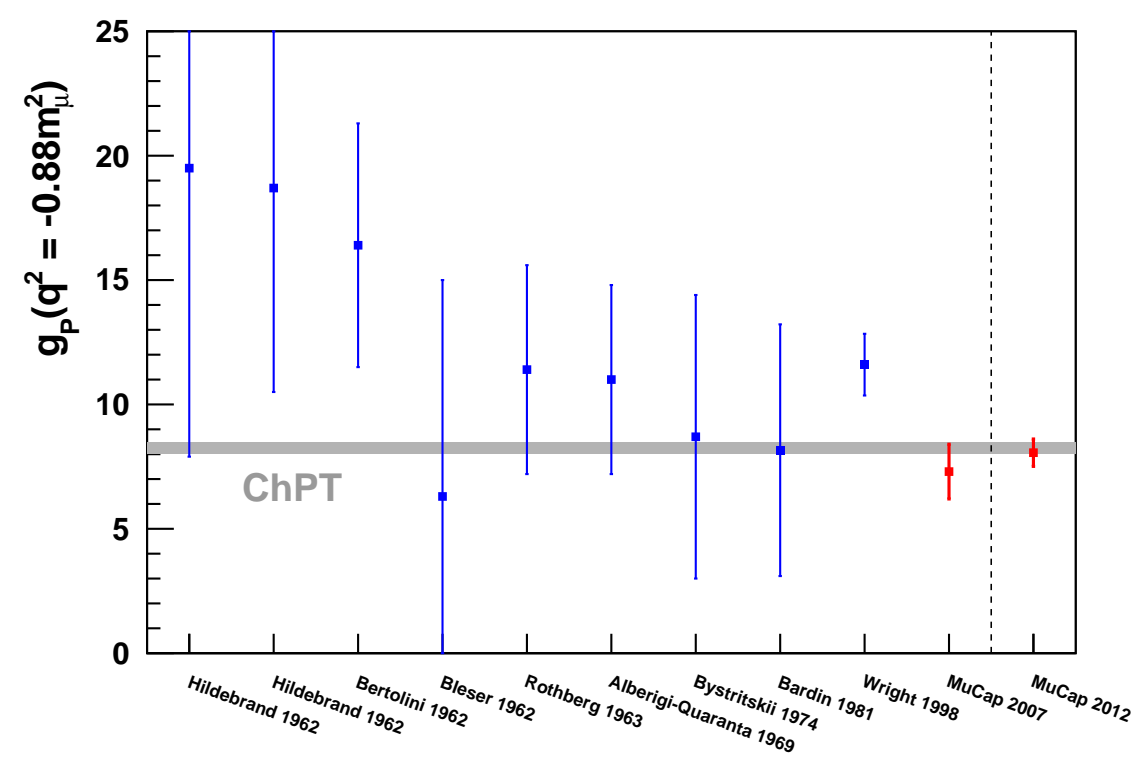

Figure 2: Experimental determinations of $g_{P}$ compared to the precise ChPT prediction. The atomic physics effects are estimated according to Ref. [1]. The relatively precise result from radiative muon capture (Wright 1998 [15]) exceeds theory. The new MuCap results (MuCap 2007 [16], MuCap 2012 [17]) are shown in red.

Muon capture on hydrogen is the most direct method to determine $g_{P}$ and experimental efforts span a period of 50 years, see Fig. 2. Experiments before MuCap only achieved limited precision and even showed some internal inconsistencies, because of several experimental challenges: i) The composition of atomic/molecular states, where muon capture occurs, has to be known, otherwise the results cannot be interpreted in terms of fundamental weak couplings. ii) Muon capture on hydrogen has a small branching ratio of typically $0.1 \%$ and leads to an all neutral final state. iii) Muon capture rates on heavier elements exceed those in hydrogen by several orders of magnitude. Thus muon stops in wall materials or transfer of muons to trace impurities in hydrogen have to be scrupulously avoided. The interplay of atomic effects, issue i), is rather unusual in nuclear/particle physics. It results from the fact that stopped muons quickly form muonic hydrogen $p \mu$, which then can combine with other hydrogen atoms to $p p \mu$ molecules, according to a rate 
$\phi \lambda_{p p}$, which is proportional to the hydrogen density $\phi$ (per convention $\phi=1$ corresponds to $\mathrm{LH}_{2}$ ). Subsequently, the $p p \mu$ molecules convert from the initial $p p \mu$ ortho-state to the para-state with a slow and poorly known rate $\lambda_{o p}$. As the capture rates from these states differ, the uncertainty in the population the molecular states induces a corresponding significant uncertainty in the interpretation of experiments with high density targets (see Fig. 3). The related, rare radiative muon capture (RMC) process $\mu^{-} p \rightarrow n v \gamma$ is less sensitive to $\lambda_{o p}$, but the first experimental result on $g_{P}$ [15] disagreed with theory. The controversial experimental situation is discussed in reviews $[1,18]$.
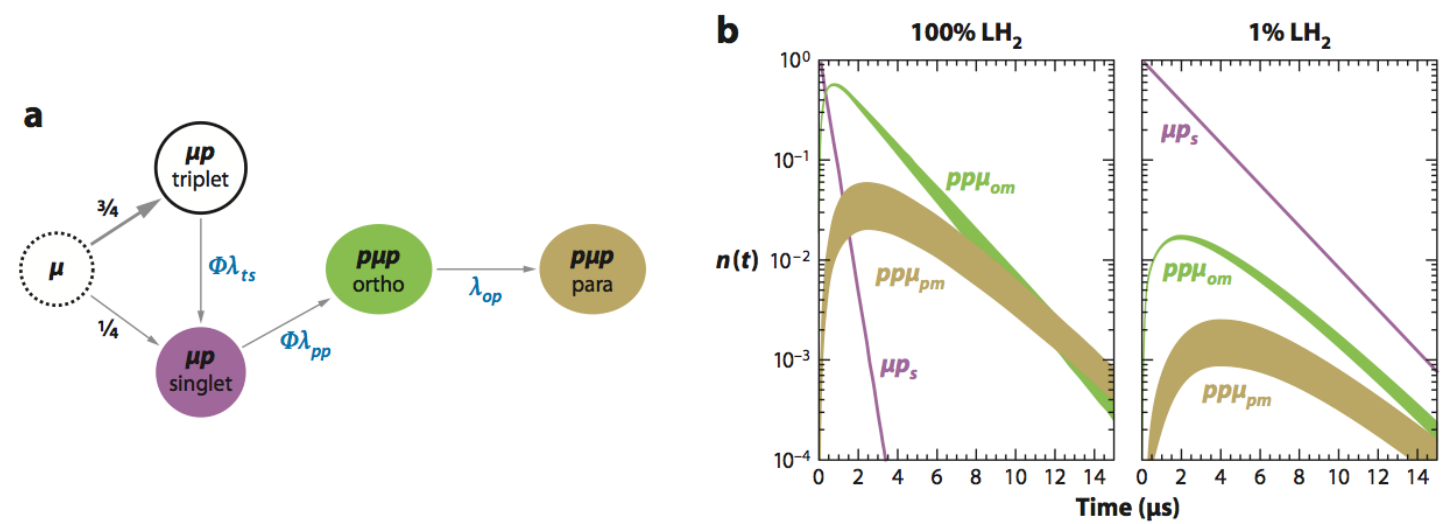

Figure 3: a) Muon-induced atomic and molecular reactions in pure hydrogen. b) Populations of $p \mu$ singlet and molecular $p p \mu$ ortho- and para states as function of time. At density $\phi=1$ the molecular states dominate, whereas at $\phi=0.01$ the muon spends $95 \%$ of its life in the singlet atomic state. Reprinted from [1].

\subsection{Weak interaction in the two-nucleon system}

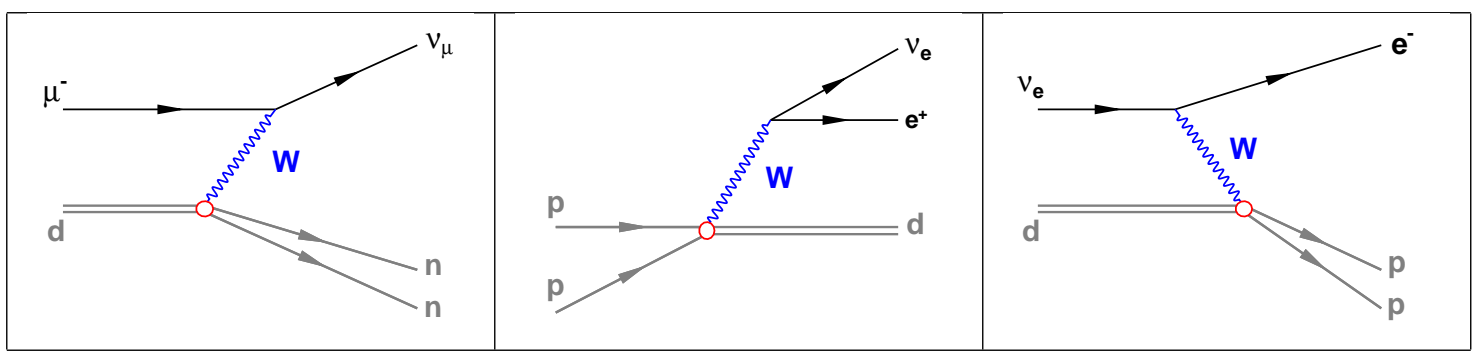

Figure 4: Feynman diagrams for the related processes $\mu^{-} d \rightarrow n n v, p p \rightarrow d e^{+} v$ and $v d \rightarrow p p e^{-}$. The red dot indicates the vertex subject to short-range hadronic physics.

As shown in Fig. 4 muon capture on the deuteron with rate $\Lambda_{D}$

$$
\mu+d \rightarrow n+n+v
$$

is closely related to electro-weak astrophysical processes of fundamental importance, whose cross sections have never been measured directly. These include $p p$ fusion, which is the primary energy source in the sun and the main sequence stars, and $v d$ reactions, which provided convincing evidence for solar neutrino oscillations at the Sudbury Neutrino Observatory. The main challenge in the calculation of these processes are the short range, two-nucleon contributions to the axial current, which currently cannot be derived from first principles. In the past, those have been modeled 
by "meson-exchange currents" (MEC), while in the framework of EFT they are expressed by low energy constants (LEC), which parametrize high momentum scale physics, that has been integrated out. For the foreseeable future, reaction (1.7) is only one of these basic weak reactions, which can be measured with precision, to determine the unknown LEC solely based on two-nucleon observables. This is the goal of the new MuSun experiment, which plans to measure $\Lambda_{D}$ to better than $1.5 \%$.

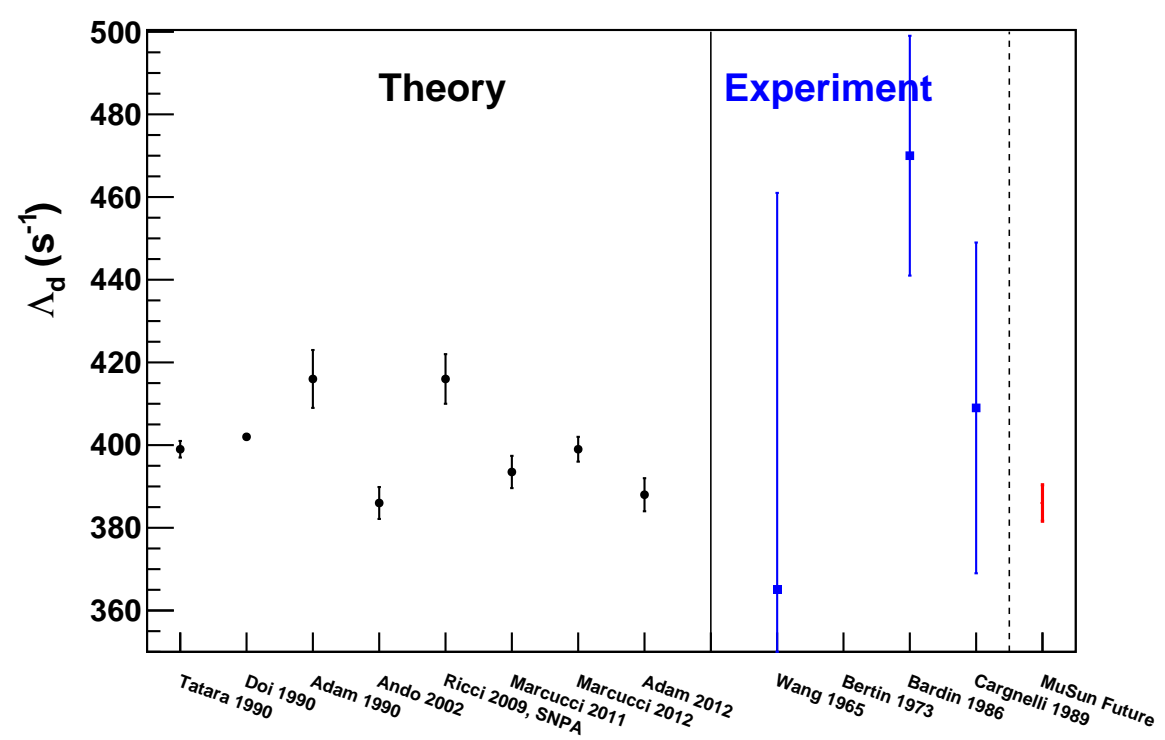

Figure 5: Left side: Recent theoretical results on muon capture rate $\Lambda_{d}$ from the doublet state of the $\mu d$ atom. The pion-less EFT calculation [19] cannot be expressed by a single value and is not included. Right side: Experimental results scatter widely (revised Bertin 1973 is off scale [1]) and most accurate result is inconsistent with modern theory. For the future MuSun result only the expected error band is indicated.

In modern calculations of the capture rate $\Lambda_{D}$, the traditional nuclear physics approach, based on the impulse approximation with explicit modeling of MECs, is being replaced by less modeldependent hybrid EFT calculations; a pion-less EFT calculation has also been performed. Recently first full EFT calculations were published [20, 21]. The theoretical program of calculating electroweak reactions from first principles is not finished yet (c.f. L. Marcucci, this conference). In particular, significant effort is expended on consistently treating the three-body forces at the same chiral order (N3LO) as the two-nucleon potentials. MuSun would provide a rather direct measure of the LECs $\hat{d}_{R}$ or $c_{D}$ on the deuteron. This will allow the verification of EFT calculation of electro-weak current interactions with two nucleons first, before expanding to three and more nucleon systems. Because of the lack of this information, existing calculations have to rely on the more complex ${ }^{3} \mathrm{He} /{ }^{3} \mathrm{H}$ system, in particular the rate of tritium beta decay, to become predictive. Unfortunately, the present experimental situation on $\mu+d$ capture is unsatisfactory. The best existing experiments $[22,23]$ are not precise enough and the most precise result differs from modern theory by three standard deviations. If true, such a discrepancy would have major ramifications for the above-mentioned astrophysics processes. In Fig. 5 theoretical results since 1990 are compared 
to all existing experimental data.

Experiments on process (1.7) face similar challenges as outlined in section 1.2 for hydrogen. Notably, the molecular physics issues enter in a different guise. The hyperfine transition rate of the upper $d \mu(\uparrow \uparrow)$ quartet to the $d \mu(\uparrow \downarrow)$ doublet state is slow. The V-A structure of weak interactions, however, disfavors capture from the quartet state compared to capture from the doublet state, so that the experimentally observed capture yield is largely proportional to the population of the doublet state. Once a $d d \mu$ molecule is formed, muon-catalyzed $d d$ fusion occurs within nanoseconds. While these fusion processes allow an accurate monitoring of the hyperfine population [24], they also generate ubiquitous fusion products, which might create significant background or distortions, depending on the experimental method employed. Moreover, the deuterium purity requirements are even more stringent than in the case of pure hydrogen. The reaction sequence is depicted in Fig. 6 and discussed in detail in review [1].

a

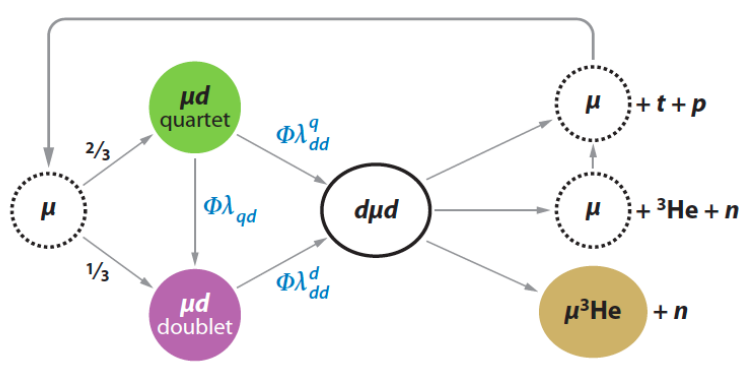

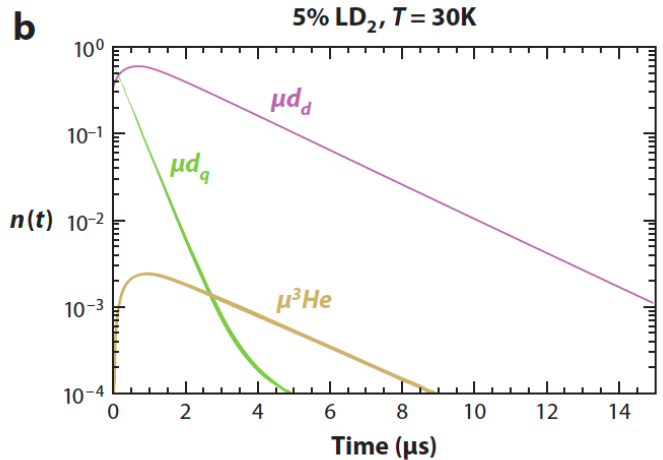

Figure 6: a) Sequence of muon-induced atomic and molecular reactions in pure deuterium. b) Populations $\mathrm{n}(\mathrm{t})$ of the two $d \mu$ hyperfine states (quartet and doublet) and the ${ }^{3} \mathrm{He} \mu$ atom at the conditions of the MuSun experiment. The density $\phi=0.05$ leads to a sufficiently fast depopulation of the quartet state, so that the muons spends $86 \%$ of its life in the doublet state. Reprinted from Ref. [1].

\section{Muon Capture on the Proton, MuCap Experiment}

Fig. 7 sketches the MuCap detector, designed according to the following strategy.

Precision technique. The experiment uses the "lifetime" technique, where the capture rate $\Lambda_{S}$ is given by the difference between the disappearance rate of negative muons in the hydrogen target and the decay rate of the free muon. The electrons from muon decay were observed with a cylindrical electron tracker, consisting of two layers of proportional chambers and a segmented scintillation hodoscope. The muon decay spectrum was constructed as the time difference between the entrance counter signals and the electron tracker.

Clear Interpretation. As demonstrated in Fig. 3b, $р p \mu$ formation can be largely suppressed, by choosing a low gas density of $\phi=0.01$. Then capture takes place predominately from the singlet $p \mu$ atom and can be interpreted unambiguously in terms of $\Lambda_{S}$.

Active target. The low density implies small hydrogen stopping mass, leading to dangerous muon stops in the target walls. These background events are rejected by tracking individual muons in an active hydrogen target. It consists of a hydrogen TPC, which was specifically developed over several years of R\&D effort [25]. 


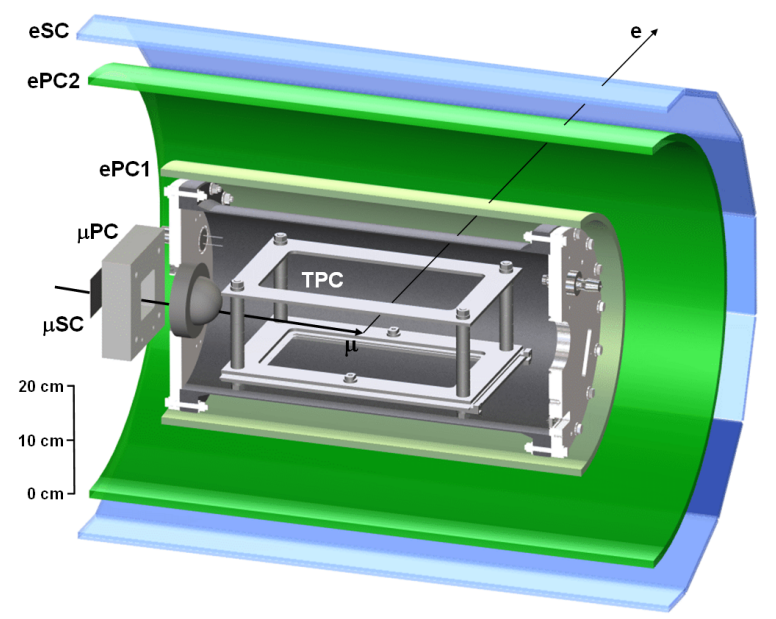

Figure 7: Cross-sectional side view of MuCap experiment.

Ultra-high chemical purity. High target purity at the $10 \mathrm{ppb}$ level was achieved by carefully selecting the TPC materials, baking the chamber and continuously circulating and filtering the gas with a non-mechanical cryogenic purification system [26]. The purity was verified by detecting charged recoils from reactions like $\mu^{-} O \rightarrow N v_{\mu}$ inside the TPC.

Record isotopic purity. Muons can transfer from hydrogen to deuterium. The so formed $d \mu$ atoms have a long free path in hydrogen and can diffuse out of the fiducial volume, distorting the observed time spectra. An on-site cryogenic distillation column was constructed, which achieved an isotopic purity of $\sim 10 \mathrm{ppb}$, as verified by accelerator mass spectroscopy.

High statistics. During each event only a single muon was allowed in the detector to avoid reconstruction ambiguities. Efficient data collection was achieved with a muon-on-request scheme, where a fast-switching electrostatic kicker turned off the beam for the measuring interval, as soon as a muon had entered the detector.

The final MuCap results are presented in Ref. [17]. A total statistics of $1.2 \times 10^{10} \mu^{-}$decays was collected, with supplemental data for systematic studies, including $\mu^{+}$and impurity doped data runs [17]. The disappearance rate of negative muons in hydrogen was consistently determined in two main production runs, including numerous consistency checks (c.f. Fig. 8).

The capture rate $\Lambda_{S}$ was determined according to $\lambda_{\mu^{-}}=\left(\lambda_{\mu^{+}}+\Delta \lambda_{\mu p}\right)+\Lambda_{S}+\Delta \Lambda_{p p \mu}$. Here $\Delta \lambda_{\mu p}$ is a calculable $\mu p$ bound-state effect, while $\Delta \Lambda_{p p \mu}$ accounts for the around $3 \%$ of muons that capture from molecular states. The result is

$$
\Lambda_{S}^{\mathrm{MuCap}}=714.9 \pm 5.4_{\text {stat }} \pm 5.1_{\text {syst }} \mathrm{s}^{-1},
$$

in excellent agreement with recent theory [27, 28, 29]. From the comparison of $\Lambda_{S}^{\mathrm{MuCap}}$ with theory, updated with the latest $g_{A}^{\mathrm{PDG}}(0)$,

$$
g_{P}^{\operatorname{MuCap}}\left(q_{0}^{2}=-0.88 m_{\mu}^{2}\right)=8.06 \pm 0.48 \pm 0.28,
$$

is extracted, where the two uncertainties arise from the error propagation from MuCap and theory, respectively. If the updated $g_{A}(0)=1.275$ would be used, $g_{P}$ would increase to 8.34. Fig. 9 summarizes the current information on $g_{P}$ based on the most accurate $\mathrm{OMC}$ result in $\mathrm{LH}_{2}$ [22], the 

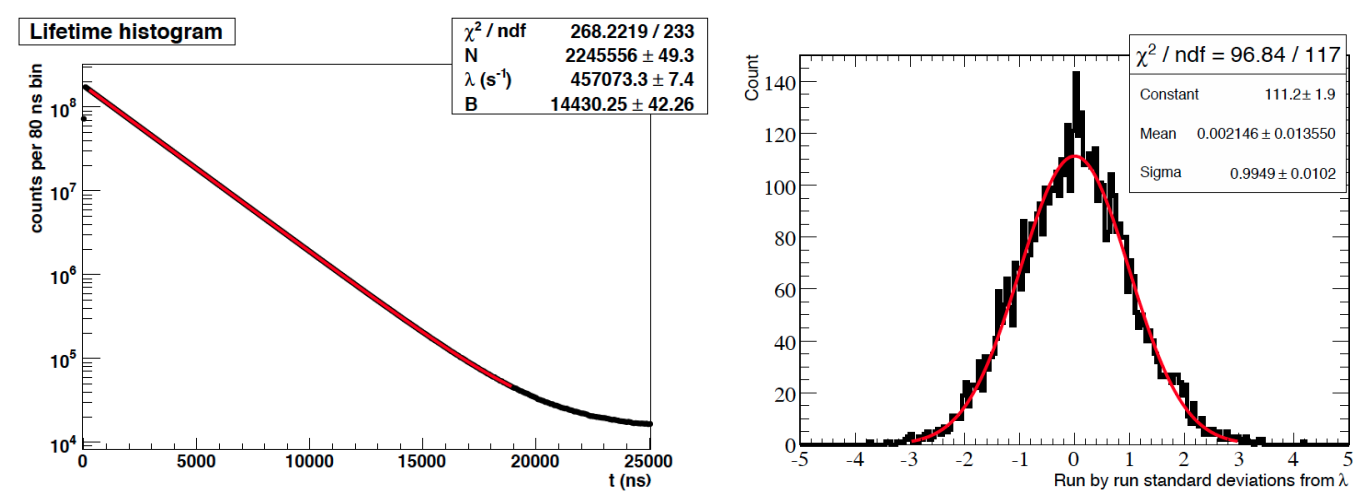

Figure 8: lhs: Exponential fit to 2006 data set, time axis still blinded, rhs: distribution of disappearance rates $\lambda$ measured over this period, from B. Kiburg, Ph.D. thesis, UIUC, 2011.

RMC measurement [15] and the new MuCap result [17]. While the earlier experimental landscape was unclear and even inconsistent, MuCap is much less sensitive to molecular uncertainties and unambiguously verifies a fundamental prediction of low-energy QCD.

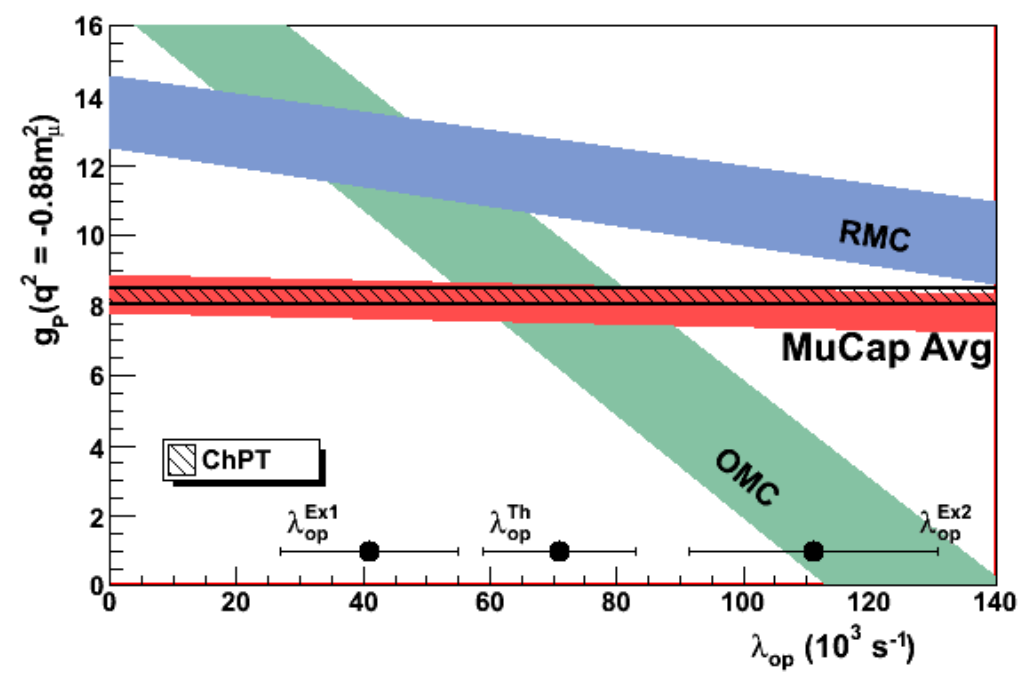

Figure 9: Extracted values for $g_{P}$ as function of the poorly known molecular transition rate $\lambda_{o p}$, from [17].

\section{Muon Capture on the Deuteron, MuSun Experiment}

Similar to MuCap, MuSun employs the "lifetime" technique and muons are tracked in an active target to select only stops in ultra pure deuterium. The muon atomic physics demands, however, a different TPC design, namely a cryogenic detector with density $\phi=0.05$. As shown in Fig. 6, the relatively high density affords fast transitions to the desired lower hyperfine state; low temperature suppresses muon-catalyzed fusion background and allows TPC operation at pressures below 10 bar. Since the target density is five times higher than that of MuCap, the chamber does not provide internal gas gain and a high drift voltages of $80 \mathrm{kV}$ are required. Excellent TPC 


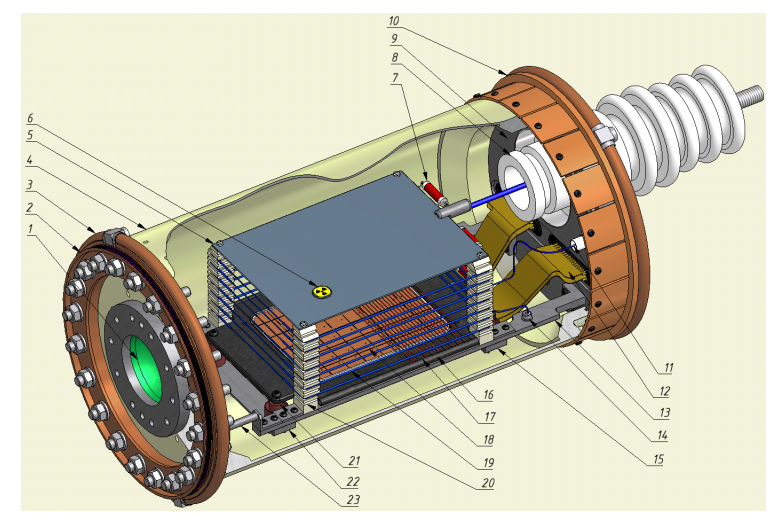

Figure 10: CryoTPC layout: 1 - beryllium window, 8 - main cathode HV feed through, 10 - heat exchangers for Ne cooling circuit, 12 - Kapton signal cables, 17 - Frisch grid, 18 - pad plane, 19 - field shaping wires.

energy resolution is critical, in particular for a determination of the muon stop location robust against fusion interference and for in - situ monitoring of impurity capture signals, which have to be discriminated against the ubiquitous ${ }^{3} \mathrm{He}$ fusion recoils. Given the small size of typically signals ( $4.4 \mathrm{fC} / \mathrm{MeV}$ ), full analog readout into FADCs and careful attention to noise sources is mandatory. This includes decoupling the acoustic oscillation induced by the cryostat and optimizing the input noise with special cryogenic preamplifiers placed in the insulation vacuum. Some features of this novel device are highlighted in Fig. 10.
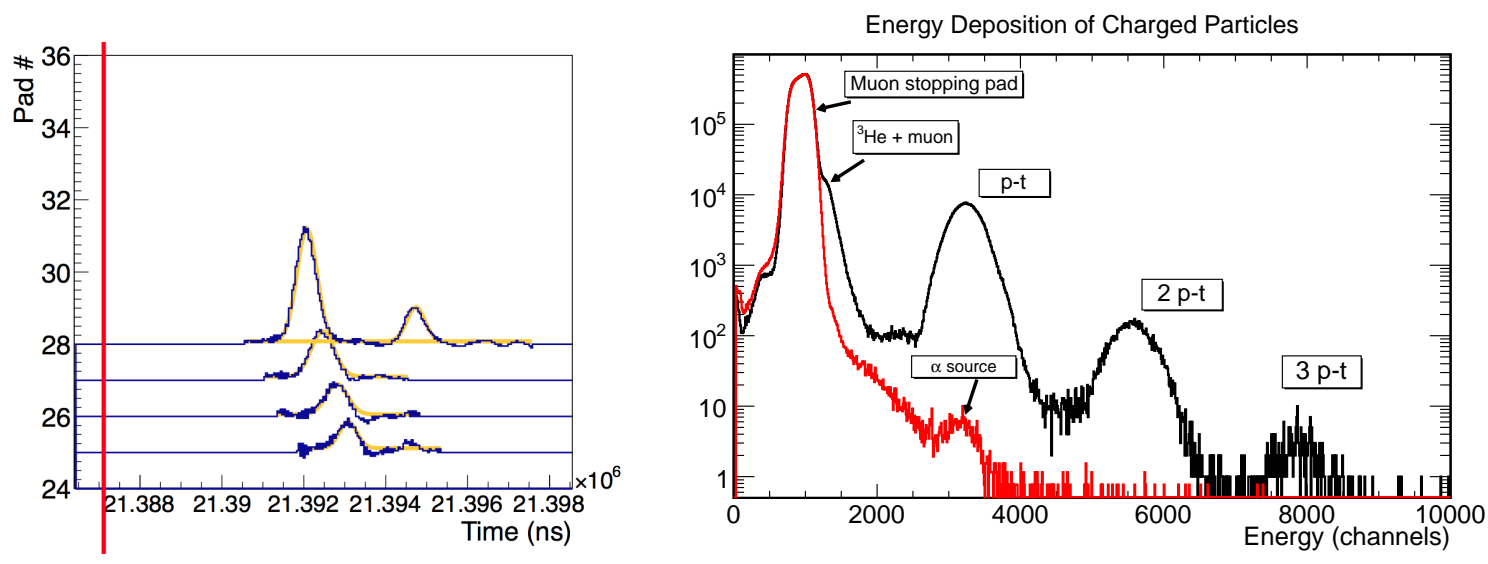

Figure 11: Left side: Sequence of muon and fusion signal on TPC pads. Right side: Energy spectra with $\mu^{-}$(black) display muon and fusion signals, whereas $\mu^{+}$(red) calibrates response to muons only.

The experiment has been commissioned and a first data run in 2011 collected about $20 \%$ of the final statistics. It is currently being analyzed towards a first physics result. Fig. 11 shows some representative data. Currently the experiment is undergoing its final upgrades and is moved into a new PSI beam line, which was commissioned this fall. Collection of the full data sets is foreseen during two long production runs in 2013 and 2014.

The MuCap and MuSun experiment were supported in part by the U.S. NSF, the U.S. DOE and CRDF, PSI, the Russian Academy of Sciences and the Russian Federation. 


\section{References}

[1] P. Kammel and K. Kubodera, Precision muon capture, Ann. Rev. Nucl. Part. Sci. 60 (2010) 327-353.

[2] T. van Ritbergen and R. G. Stuart, Complete 2-loop quantum electrodynamic contributions to the muon lifetime in the Fermi model, Phys. Rev. Lett. 82 (1999) 488-491, [hep-ph/9808283].

[3] MuLan Collaboration, D. B. Chitwood et al., Improved Measurement of the Positive Muon Lifetime and Determination of the Fermi Constant, Phys. Rev. Lett. 99 (2007) 032001, [0 704.1981 ].

[4] MuLan Collaboration, D. Webber et al., Measurement of the Positive Muon Lifetime and Determination of the Fermi Constant to Part-per-Million Precision, Phys. Rev. Lett. 106 (2011) 041803.

[5] MuLan Collaboration, V. Tishchenko et al., Detailed report of the MuLan measurement of the positive muon lifetime and determination of the Fermi constant, Phys. Rev. D 87 (2013) 052003.

[6] Particle Data Group Collaboration, J. Beringer et al., Review of Particle Physics (RPP), Phys.Rev. D86 (2012) 010001.

[7] E. Blucher and W. Marciano. $V_{u d}, V_{u s}$, The Cabibbo Angle and CKM Unitarity, in J. Beringer et. al (Particle Data Group), Phys. Rev. D86, 010001 (2012), 852.

[8] D. Mund, B. Maerkisch, M. Deissenroth, J. Krempel, M. Schumann, et al., Determination of the Weak Axial Vector Coupling from a Measurement of the Beta-Asymmetry Parameter A in Neutron Beta Decay, arXiv:1204.0013.

[9] UCNA Collaboration, M. Mendenhall et al., Precision Measurement of the Neutron Beta-Decay Asymmetry, arXiv:1210.7048.

[10] V. Bernard, L. Elouadrhiri, and U. G. Meissner, Axial structure of the nucleon, J. Phys. G28 (2002) R1-R35, [hep-ph/0107088].

[11] V. Bernard, N. Kaiser, and U.-G. Meissner, QCD accurately predicts the induced pseudoscalar coupling constant, Phys. Rev. D50 (1994) 6899-6901, [hep-ph/9403351].

[12] N. Kaiser, Induced pseudoscalar form factor of the nucleon at two-loop order in chiral perturbation theory, Phys. Rev. C67 (2003) 027002, [nucl-th/ 0301034 ].

[13] RBC and UKQCD Collaboration, T. Yamazaki et al., Nucleon form factors with $2+1$ flavor dynamical domain-wall fermions, Phys. Rev. D 79 (2009) 114505.

[14] C. Alexandrou et al., Axial nucleon form factors from lattice QCD, Phys. Rev. D 83 (2011) 045010.

[15] D. H. Wright et al., Measurement of the induced pseudoscalar coupling using radiative muon capture on hydrogen, Phys. Rev. C57 (1998) 373-390.

[16] MuCap Collaboration, V. A. Andreev et al., Measurement of the Rate of Muon Capture in Hydrogen Gas and Determination of the Proton's Pseudoscalar Coupling $g_{P}$, Phys. Rev. Lett. 99 (2007) 032002.

[17] MuCap Collaboration, V. Andreev et al., Measurement of Muon Capture on the Proton to 1\% Precision and Determination of the Pseudoscalar Coupling $g_{P}$, Phys.Rev.Lett. 110 (2013) 012504.

[18] T. Gorringe and H. W. Fearing, Induced pseudoscalar coupling of the proton weak interaction, Rev. Mod. Phys. 76 (2004) 31-91, [nucl-th/ 0206039 ].

[19] J.-W. Chen, T. Inoue, X.-d. Ji, and Y.-C. Li, Fixing Two-Nucleon Weak-Axial Coupling $L_{1, A}$ From $\mu^{-} \mathrm{d}$ Capture, Phys. Rev. C 72 (2005) 061001, [nucl-th/ 0506001 ]. 
[20] L. Marcucci, A. Kievsky, S. Rosati, R. Schiavilla, and M. Viviani, Chiral effective field theory predictions for muon capture on deuteron and ${ }^{3}$ He, Phys.Rev.Lett. 108 (2012) 052502.

[21] J. Adam, J., M. Tater, E. Truhlik, E. Epelbaum, R. Machleidt, et al., Calculation of Doublet Capture Rate for Muon Capture in Deuterium within Chiral Effective Field Theory, Phys. Lett. B 709 (2012) 93-100, [arXiv:1110.3183].

[22] G. Bardin et al., A measurement of the rate of muon capture in liquid deuterium by the lifetime technique, Nucl. Phys. A 453 (1986) 591.

[23] M. Cargnelli et al., Measurement of the muon capture rate in gaseous deuterium, in Proceedings of the XXIII Yamada Conf. on Nuclear Weak Processes and Nuclear Structure, Osaka, Japan, 1989.

[24] W. H. Breunlich, P. Kammel, J. S. Cohen, and M. Leon, Muon-catalyzed fusion, Ann. Rev. Nucl. Part. Sci. 39 (1989) 311-356.

[25] MuCap Collaboration, J. Egger, M. Hildebrandt, and C. Petitjean, The 10bar hydrogen time projection chamber of the MuCap experiment, Nucl.Instrum.Meth. A628 (2011) 199-203.

[26] V. A. Ganzha et al., A circulating hydrogen ultra-high purification system for the MuCap experiment, Nucl. Instrum. Meth. A578 (2007) 485-497, [0 05 . 1473].

[27] V. Bernard, T. R. Hemmert, and U.-G. Meissner, Ordinary and radiative muon capture on the proton and the pseudoscalar form factor of the nucleon, Nucl. Phys. A686 (2001) 290-316.

[28] S. Ando, F. Myhrer, and K. Kubodera, Capture rate and neutron helicity asymmetry for ordinary muon capture on hydrogen, Phys. Rev. C63 (2001) 015203, [nucl-th/ 0008003 ].

[29] A. Czarnecki, W. J. Marciano, and A. Sirlin, Electroweak radiative corrections to muon capture, Phys. Rev. Lett. 99 (Jul, 2007) 032003. 